\title{
Some remarks on the performance of Matlab, Python and Octave in simulating dynamical systems
}

\author{
Priscila F. S. Guedes, ${ }^{*}$ Erivelton G. Nepomuceno ${ }^{* *}$ \\ * Graduate Program in Electrical Engineering - Universidade Federal \\ de Minas Gerais - Av. Antônio Carlos 6627, 31270-901, Belo \\ Horizonte, MG,Brazil (e-mail: pri12_guedes@hotmail.com). \\ ** Control and Modelling Group (GCOM), Department of Electrical \\ Engineering, Federal University of São João del-Rei, São João del-Rei, \\ MG,36307-352, Brazil b (e-mail: nepomuceno@ufsj.edu.br)
}

\begin{abstract}
Matlab has been considered as a leader computational platform for many engineering fields. Well documented and reliable, Matlab presents as a great advantage its ability to increase the user productivity. However, Python and Octave are among some of the languages that have challenged Matlab. Octave and Python are well known examples of high-level scripting languages, with a great advantage of being open source software. The novelty of this paper is devoted to offer a comparison among these tree languages in the simulation of dynamical systems. We have applied the lower bound error to estimate the error of simulation. The comparison was performed with the chaotic systems Duffing-Ueda oscillator and the Chua's circuit, both identified with polynomial NARMAX. Octave presents the best reliable outcome. Nevertheless, Matlab needs the lowest time to undertake the same activity. Python has presented the worse result for the stop simulation criterion.
\end{abstract}

Keywords: Matlab; Octave; Python; Chaos; Lower Bound Error; Dynamical Systems; Computer Arithmetic.

\section{INTRODUCTION}

Computational simulations are fundamental in analysis of nonlinear dynamical systems Galias (2013); Lozi (2013); Sauer et al. (1997); Hammel et al. (1987). Among the used softwares, Matlab stands out due to its high performance oriented to the numerical calculation. Additionally, Matlab has been used to develop many well-cited toolbox to control theory, such as YALMIP Lofberg (2004) or System Identification Toolbox Ljung et al. (2009). Octave is free software that is more compatible with Matlab. And in recent years, Python software has become popular and its popularity is also ranked first in the IEEE Spectrum ranking for 2018 IEEE Spectrum (2018). The three software are examples of scripting languages, that is, these languages are interpreted.

These softwares are used in digital computers that have inherent properties which numerical simulations do not present exact results Galias (2013). This fact occurs due to the representation of the real number on computer, which may cause approximations, rounding and truncation errors Liao (2009). Error propagation control is considered highly important, especially when they characterize chaotic systems, since small errors introduced in each computational step can grow exponentially due to the high sensitivity presented in chaotic systems Mendes and Nepomuceno (2016); Nepomuceno and Martins (2016). In addition to

\footnotetext{
* This work has been supported by the Brazilian agency CAPES.
}

rounding errors, truncation errors are introduced during integration of continuous time systems by using numerical methods, which are constructed by skipping higher-order terms in the Taylor expansion of the solution, these errors decrease computational reliability Qin and Liao (2018).

Lozi (2013) states that there are several published works related to chaotic dynamical systems, which the results were not carefully checked, compromising the reliability of the results. In this context, Nepomuceno (2014) showed that the iterations of the Logistic Map generated a result that mathematically was not what was expected. In addition, the use of different discretization methods results in different numerical solutions Liao (2009); Nepomuceno and Mendes (2017).

In order to investigate numerical error in system simulations, Nepomuceno et al. (2017) proposed a method to calculate the Lower Bound Error (LBE) based on the fact that two equivalent mathematical extensions can generate divergent results on computational simulations. Based on this method there was an expansion to an arbitrary number of mathematical extensions Guedes et al. (2017); Chaves et al. (2006); Unpingco (2008); de la Fraga et al. (2017). In this sense, few studies have considered the influence of the programming language on the reliability of numerical solutions Junior et al. (2017). Usually the comparison of such languages has been devoted to time consumption or arithmetic and algebraic operations, as done by Unpingco (2008). The novelty of this paper is 
devoted to offer a comparison among these tree languages in the simulation of dynamical systems.

The rest of the paper is organized as follows. In Section 2 we recall some preliminary concepts. Then, in Section 3 , we present the developed method. Section 4 is devoted to present the results, then the final remarks are given in Section 5 .

\section{PRELIMINARY CONCEPTS}

\subsection{The polynomial NARMAX}

The NARMAX model is a representation for nonlinear systems. This model can be represented as Chen and Billings (1989)

$$
\begin{aligned}
y(k)= & F^{l}\left[y(k-1), \cdots, y\left(k-n_{y}\right),\right. \\
& u(k-1), \cdots, u\left(k-n_{u}\right), \\
& \left.e(k-1), \cdots, e\left(k-n_{e}\right)\right]+e(k),
\end{aligned}
$$

where $y(k), u(k)$ e $e(k)$ are, respectively, the output, the input and the noise terms at the discrete time $n \in \mathbb{N}$. The parameters $n_{y}, n_{u}$ e $n_{e}$ are their maximum delay. And $F^{\ell}$ is a nonlinear function of degree $\ell$.

\subsection{Recursive functions}

In recursive functions is possible to calculate the state $x_{n+1}$, at a give time, from an earlier state $x_{n}$

$$
x_{n+1}=f\left(x_{n}\right),
$$

where $f$ is a recursive function and $x_{n}$ is a function state at the discrete time $n$. Given an initial condition $x_{0}$ and with successive applications of the function $f$ it is possible to know the sequence $x_{n}$ Gilmore and Lefranc (2012).

\subsection{Natural interval extension}

The natural interval extension is achieved by changing the sequence of arithmetic operation Moore et al. (2009), that is, the extensions are mathematically equivalents.

Furthermore, two extension which algebraically are the same function may not be equivalent in interval arithmetic

Example 2.3: Based on the logistic map May (1976), interval extensions are:

$$
\begin{array}{r}
x_{n+1}=r x_{n}\left(1-x_{n}\right) \\
x_{n+1}=r x_{n}-r x_{n}^{2} \\
x_{n+1}=r x_{n}-r x_{n} x_{n}
\end{array}
$$

\subsection{Orbits and pseudo-orbits}

Associated with a map we may define an orbit as follows Hammel et al. (1987):

Definição 1. The true orbit $\left\{x_{n}\right\}_{n=0}^{N}$ satisfies $x_{n+1}=$ $f\left(x_{n}\right)$.

That is, given an initial condition $x_{0}$, and interacting the function, a sequence of values represented by $\left\{x_{n}\right\}=$ $\left[x_{0}, x_{1}, \cdots, x_{n}\right]$ is defined. When the computer is used to calculate the recursive functions, numeric errors are propagated during successive calculations, then the true orbit is not calculated but a representation of the same, which is called pseudo-orbit

$$
\left\{\hat{x}_{i, n}\right\}=\left[\hat{x}_{i, 0}, \hat{x}_{i, 1}, \cdots, \hat{x}_{i, n}\right],
$$

which accepts the relation

$$
\left|x_{n}-\hat{x}_{i, n}\right| \leq \delta_{i, n}
$$

where $\delta_{i, n} \in \mathbb{R}^{+}$.

Thus, we define an interval associated with each value of a pseudo-orbit

$$
I_{i, n}=\left[\hat{x}_{i, n}-\delta_{i, n}, \hat{x}_{i, n}+\delta_{i, n}\right] .
$$

From Equations (4) and (5) it is clear that

$$
x_{n} \in I_{i, n} \quad \text { for all } \mathrm{i} \in \mathbb{N} \text {. }
$$

\subsection{The lower bound error}

The lower bound error consists of a tool to analyze the error propagation in numerical simulations proposed by Nepomuceno et al. (2017).

Theorem 1. Let two pseudo-orbits $\left\{\hat{x}_{a, n}\right\}$ and $\left\{\hat{x}_{b, n}\right\}$ derived from two natural interval extensions. Let $\ell_{\Omega, n}=$ $\left|\hat{x}_{a, n}-\hat{x}_{b, n}\right| / 2$ be the lower bound error associated to the set of pseudo-orbits $\Omega=\left[\left\{\hat{x}_{a, n}\right\},\left\{\hat{x}_{b, n}\right\}\right]$ of a map, then $\gamma_{a, n}=\gamma_{b, n} \geq \ell_{\Omega, n}$.

The proof of this theorem can be found in Nepomuceno et al. (2017).

\section{METHODS}

Nepomuceno et al. (2017) developed the Lower Bound Error theorem. But, different software can cause different result. Hence, the objective is to investigate and compare the Matlab R2016a-64 bits, Octave 4.2.1 - 64 bits and Python 3.5.4-64 bits performances using the Theorem 1 and the polynomial NARMAX for the Duffing-Ueda oscillator and Chua's circuit.

The proposed method can be summarized in the following steps:

(1) Step 1: choose two natural interval extensions for each system;

(2) Step 2: calculate the system's orbit from the chosen extensions in each software;

(3) Step 3: determine the lower bound error;

(4) Step 4: compare the results obtained in each software. This parallel will be performed using a stop simulation criterion and verifying in how many iterations each software reached that criterion. And also by the time each software spends to simulate the algorithms.

\subsection{Stop simulation criterion}

It was used a method which verifies the loss of simulation accuracy as a stop criterion. Then, $\varepsilon_{\alpha, n}$ is the relative precision at iteration $n$ defined by

$$
\varepsilon_{\alpha, n}=\frac{\hat{x}_{a, n}-\hat{x}_{b, n}}{\hat{x}_{a, n}+\hat{x}_{b, n}}
$$


where $n \in \mathbb{N}, \hat{x}_{a, n}$ and $\hat{x}_{b, n}$ are the two chosen pseudoorbits $a$ and $b$. A minimum precision, $\varepsilon$, is defined, which implies that the simulation would be stopped at the moment when $\varepsilon_{\alpha, n}>\varepsilon$. In this work, we adopt $\varepsilon=0.001$.

To perform the tests, it was used a computer with a processor Intel Core i5-3317U @ $1.7 \mathrm{GHz}$ and a Windows 10 Home Single Language operating system. All data, routines and simulations used in this work are available upon request.

\section{NUMERICAL EXPERIMENTS}

\subsection{Duffing-Ueda}

Considering a damped, periodically forced nonlinear DuffingUeda oscillator Billings (2013):

$$
\frac{d^{2} y}{d t^{2}}+k \frac{d y}{d t}+\mu y^{3}=A \cos (t)
$$

where $\mu$ is the cubic stiffness parameter, $k$ is a linear damping and $A$ is the amplitude of excitation. A polynomial NARMAX for the Duffing-Ueda oscillator was identified by Aguirre and Billings (1994).

$$
\begin{aligned}
\mathrm{y}_{\mathrm{n}+1}= & 2.1579 \mathrm{y}_{\mathrm{n}}-1.3203 \mathrm{y}_{\mathrm{n}-1}+0.16239 \mathrm{y}_{\mathrm{n}-2} \\
& +0.0003416 \mathrm{u}_{\mathrm{n}}+0.001963 \mathrm{u}_{\mathrm{n}-1} \\
& -0.0048196 \mathrm{y}_{\mathrm{n}}^{3}+0.003523 \mathrm{y}_{\mathrm{n}}^{2} \mathrm{y}_{\mathrm{n}-1} \\
& -0.0012162 \mathrm{y}_{\mathrm{n}} \mathrm{y}_{\mathrm{n}-1} \mathrm{y}_{\mathrm{n}-2}+0.0002248 \mathrm{y}_{\mathrm{n}-2}^{3}
\end{aligned}
$$

where $u=A \cos \left(k T_{s}\right), n \in \mathbb{N}$ and $T_{s}=\pi / 60$.

Let us consider two interval extensions of the model 9:

$$
\begin{aligned}
\mathrm{F}\left(\mathrm{X}_{\mathrm{n}}\right)= & 2.1579 \mathrm{X}_{\mathrm{n}}-1.3203 \mathrm{X}_{\mathrm{n}-1}+0.16239 \mathrm{X}_{\mathrm{n}-2} \\
& +0.0003416 \mathrm{U}_{\mathrm{n}}+0.001963 \mathrm{U}_{\mathrm{n}-1} \\
& \frac{-0.0048196 \mathrm{X}_{\mathrm{n}}^{3}+0.003523 \mathrm{X}_{\mathrm{n}}^{2} \mathrm{X}_{\mathrm{n}-1}}{-0.0012162 \mathrm{X}_{\mathrm{n}} \mathrm{X}_{\mathrm{n}-1} \mathrm{X}_{\mathrm{n}-2}+0.0002248 \mathrm{X}_{\mathrm{n}-2}^{3}} \\
\mathrm{G}\left(\mathrm{X}_{\mathrm{n}}\right)= & 2.1579 \mathrm{X}_{\mathrm{n}}-1.3203 \mathrm{X}_{\mathrm{n}-1}+0.16239 \mathrm{X}_{\mathrm{n}-2} \\
& +0.0003416 \mathrm{U}_{\mathrm{n}}+0.001963 \mathrm{U}_{\mathrm{n}-1} \\
& -0.0048196 \mathrm{X}_{\mathrm{n}}^{2} \mathrm{X}_{\mathrm{n}}+0.003523 \mathrm{X}_{\mathrm{n}}^{2} \mathrm{X}_{\mathrm{n}-1} \\
& -0.0012162 \mathrm{X}_{\mathrm{n}} \mathrm{X}_{\mathrm{n}-1} \mathrm{X}_{\mathrm{n}-2}+0.0002248 \mathrm{X}_{\mathrm{n}-2}^{3}
\end{aligned}
$$

Figure 1 shows the free-run simulation for Duffing-Ueda oscillator. For this system, it can be observed that running different software cause a different pseudo-orbit. Figure 2 shows the evolution of the Lower Bound Error. Analyzing the number of iterations that satisfies $\varepsilon=0.001$, the simulation is no longer reliable when $n \geq 4450$ for Python, $n \geq 4471$ for Matlab and $n \geq 5258$ for Octave. Therefore, Matlab represents a $0.47 \%$ greater confidence compared to Python and Octave a $18.16 \%$ greater confidence to Python.

\subsection{Chua's circuit}

The Chua's circuit (see Figure 3) Chua et al. (1993) is composed of passive linear elements (two capacitors, one inductor and one resistor) connected to an active nonlinear component, known as the Chua's diode. The Chua's circuit

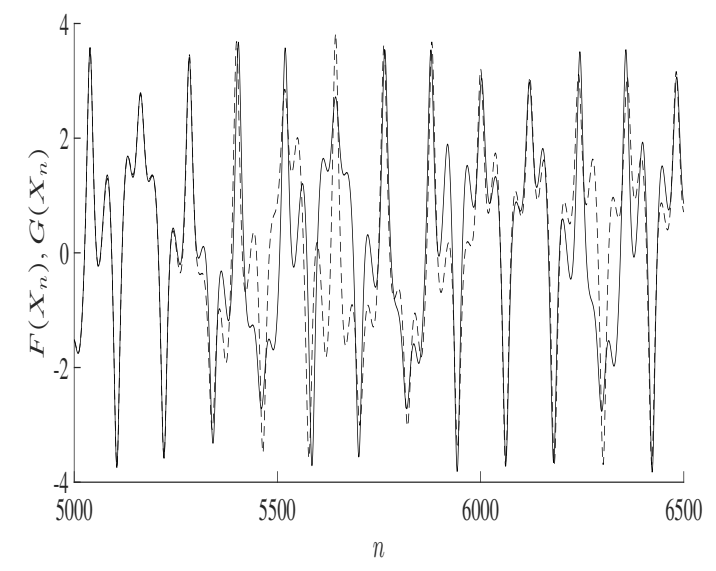

(a) Pseudo-orbits obtained from Python.

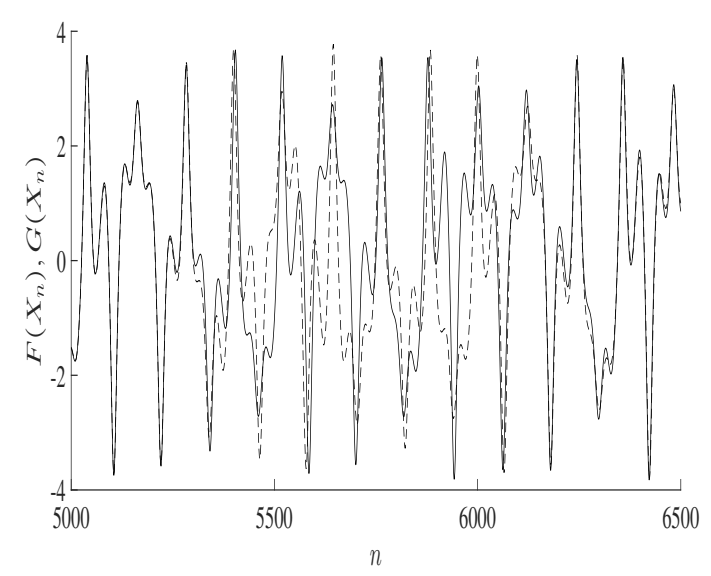

(b) Pseudo-orbits obtained from Matlab.

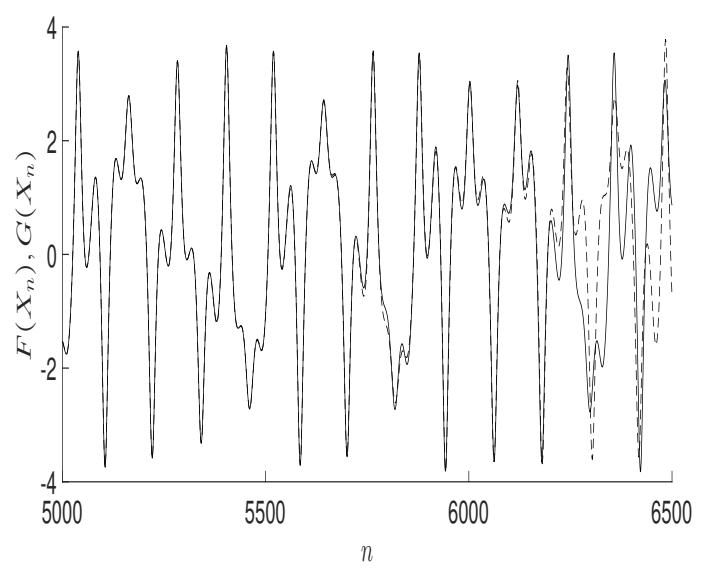

(c) Pseudo-orbits obtained from Octave.

Figure 1. Duffing-Ueda Oscillator: Free-run simulation for the interval extensions of Equations (10) and (11), with results for $F\left(X_{n}\right)(-)$ and $G\left(X_{n}\right)(--)$ and $n$ stands for the number of iterations.

is able to reproduce different regimes, such as periodic and chaotic oscillations. Its equations are described as follows. 


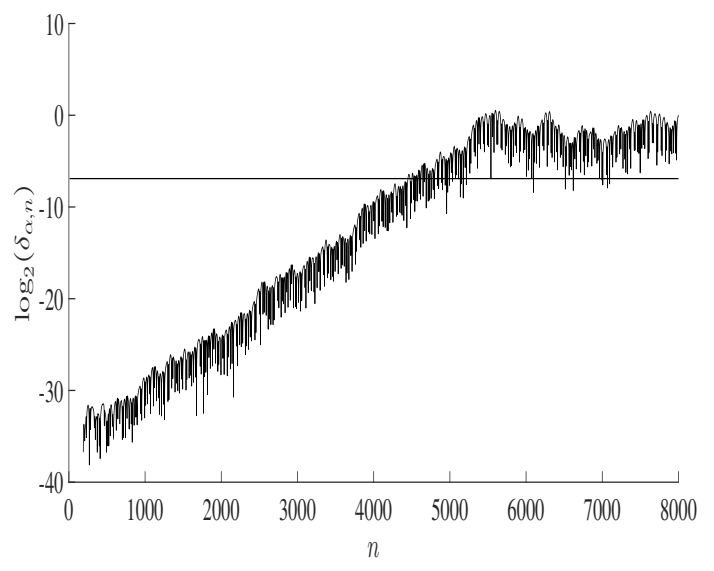

(a) LBE obtained from Python.

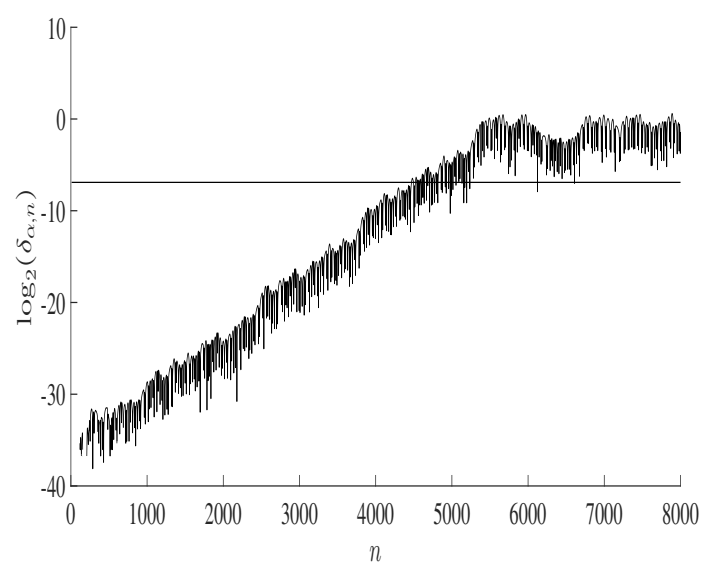

(b) LBE obtained from Matlab.

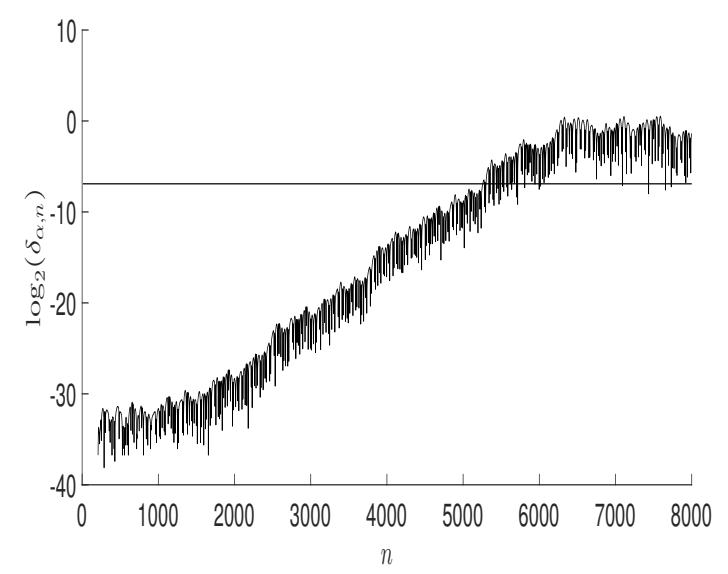

(c) LBE obtained from Octave.

Figure 2. Duffing-Ueda Oscillator: Evolution of Lower Bound Error $\ell_{\Omega, n}$. The values are plotted using $\log _{2}$.

$$
\left\{\begin{aligned}
C_{1} \frac{d v_{c_{1}}}{d t} & =\frac{v_{c_{2}}-v_{c_{1}}}{R}-i_{R}\left(v_{c_{1}}\right) \\
C_{2} \frac{d v_{c_{2}}}{d t} & =\frac{v_{c_{1}}-v_{c_{2}}}{R}+i_{L} \\
L \frac{d i_{L}}{d t} & =-v_{c_{2}}
\end{aligned}\right.
$$

The current through the nonlinear element, $i_{R}\left(v_{C_{1}}\right)$ is given by equation (13):

$$
i_{R}\left(v_{c_{1}}\right)= \begin{cases}m_{0} v_{1}+B_{p}\left(m_{0}-m_{1}\right) & v_{c_{1}}<-B_{p} \\ m_{1} v_{1} & \left|v_{c_{1}}\right| \leq B_{p} \\ m_{0} v_{1}+B_{p}\left(m_{1}-m_{0}\right) & v_{c_{1}}>-B_{p}\end{cases}
$$

where $m_{0}, m_{1}$ and $B_{p}$ are the slopes and the breaking points of the nonlinear element, respectively.

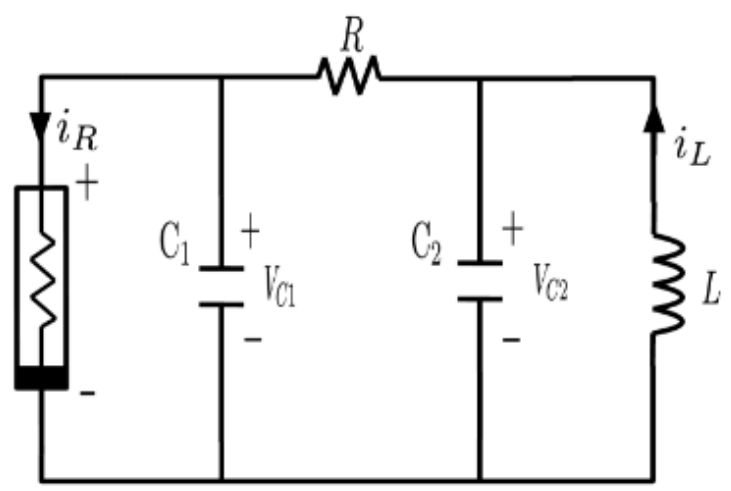

Figure 3. Chua's circuit.

A polynomial NARMAX identified for Chua's circuit is given by Aguirre (1997).

$$
\begin{aligned}
\mathrm{y}_{\mathrm{n}+1}= & 3.523 \mathrm{y}_{\mathrm{n}}-4.2897 \mathrm{y}_{\mathrm{n}-1}-0.2588 \mathrm{y}_{\mathrm{n}-3} \\
& -1.7784 \mathrm{y}_{\mathrm{n}}^{3}+2.0652 \mathrm{y}_{\mathrm{n}-2}+6.1761 \mathrm{y}_{\mathrm{n}}^{2} \mathrm{y}_{\mathrm{n}-1} \\
& +0.1623 \mathrm{y}_{\mathrm{n}} \mathrm{y}_{\mathrm{n}-1} \mathrm{y}_{\mathrm{n}-3}-2.7381 \mathrm{y}_{\mathrm{n}}^{2} \mathrm{y}_{\mathrm{n}-2} \\
& -5.5369 \mathrm{y}_{\mathrm{n}} \mathrm{y}_{\mathrm{n}-1}^{2}+0.1031 \mathrm{y}_{\mathrm{n}-1}^{3}+0.4623 \mathrm{y}_{\mathrm{n}-3}^{3} \\
& -0.5247 \mathrm{y}_{\mathrm{n}-1}^{2} \mathrm{y}_{\mathrm{n}-3}-1.8965 \mathrm{y}_{\mathrm{n}} \mathrm{y}_{\mathrm{n}-2}^{2} \\
& +5.4255 \mathrm{y}_{\mathrm{n}} \mathrm{y}_{\mathrm{n}-1} \mathrm{y}_{\mathrm{n}-2}+0.7258 \mathrm{y}_{\mathrm{n}-1} \mathrm{y}_{\mathrm{n}-3}^{2} \\
& -1.7684 \mathrm{y}_{\mathrm{n}-2} \mathrm{y}_{\mathrm{n}-3}^{2}+1.1800 \mathrm{y}_{\mathrm{n}-2}^{2} \mathrm{y}_{\mathrm{n}-3},
\end{aligned}
$$

where the time interval between $n$ and $n+1$ is $12 \mu \mathrm{s}$.

Considering two natural interval extensions of model 14 , we have

$$
\begin{aligned}
\mathrm{F}\left(\mathrm{X}_{\mathrm{n}}\right)= & 3.523 \mathrm{X}_{\mathrm{n}}-4.2897 \mathrm{X}_{\mathrm{n}-1}-0.2588 \mathrm{X}_{\mathrm{n}-3} \\
& -1.7784 \mathrm{X}_{\mathrm{n}}^{3}+2.0652 \mathrm{X}_{\mathrm{n}-2}+\frac{6.1761 \mathrm{X}_{\mathrm{n}}^{2} \mathrm{X}_{\mathrm{n}-1}}{} \\
& +0.1623 \mathrm{X}_{\mathrm{n}} \mathrm{X}_{\mathrm{n}-1} \mathrm{X}_{\mathrm{n}-3}-2.7381 \mathrm{X}_{\mathrm{n}}^{2} \mathrm{X}_{\mathrm{n}-2} \\
& -5.5369 \mathrm{X}_{\mathrm{n}} \mathrm{X}_{\mathrm{n}-1}^{2}+0.1031 \mathrm{X}_{\mathrm{n}-1}^{3}+0.4623 \mathrm{X}_{\mathrm{n}-3}^{3} \\
& -0.5247 \mathrm{X}_{\mathrm{n}-1}^{2} \mathrm{X}_{\mathrm{n}-3}-1.8965 \mathrm{X}_{\mathrm{n}} \mathrm{X}_{\mathrm{n}-2}^{2} \\
& +5.4255 \mathrm{X}_{\mathrm{n}} \mathrm{X}_{\mathrm{n}-1} \mathrm{X}_{\mathrm{n}-2}+0.7258 \mathrm{X}_{\mathrm{n}-1} \mathrm{X}_{\mathrm{n}-3}^{2} \\
& -1.7684 \mathrm{X}_{\mathrm{n}-2} \mathrm{X}_{\mathrm{n}-3}^{2}+1.1800 \mathrm{X}_{\mathrm{n}-2}^{2} \mathrm{X}_{\mathrm{n}-3},
\end{aligned}
$$




$$
\begin{aligned}
\mathrm{G}\left(\mathrm{X}_{\mathrm{n}}\right)= & 3.523 \mathrm{X}_{\mathrm{n}}-4.2897 \mathrm{X}_{\mathrm{n}-1}-0.2588 \mathrm{X}_{\mathrm{n}-3} \\
& -1.7784 \mathrm{X}_{\mathrm{n}}^{3}+2.0652 \mathrm{X}_{\mathrm{n}-2}+\frac{6.1761 \mathrm{X}_{\mathrm{n}} \mathrm{X}_{\mathrm{n}} \mathrm{X}_{\mathrm{n}-1}}{} \\
& +0.1623 \mathrm{X}_{\mathrm{n}} \mathrm{X}_{\mathrm{n}-1} \mathrm{X}_{\mathrm{n}-3}-2.7381 \mathrm{X}_{\mathrm{n}}^{2} \mathrm{X}_{\mathrm{n}-2} \\
& -5.5369 \mathrm{X}_{\mathrm{n}} \mathrm{X}_{\mathrm{n}-1}^{2}+0.1031 \mathrm{X}_{\mathrm{n}-1}^{3}+0.4623 \mathrm{X}_{\mathrm{n}-3}^{3} \\
& -0.5247 \mathrm{X}_{\mathrm{n}-1}^{2} \mathrm{X}_{\mathrm{n}-3}-1.8965 \mathrm{X}_{\mathrm{n}} \mathrm{X}_{\mathrm{n}-2}^{2} \\
& +5.4255 \mathrm{X}_{\mathrm{n}} \mathrm{X}_{\mathrm{n}-1} \mathrm{X}_{\mathrm{n}-2}+0.7258 \mathrm{X}_{\mathrm{n}-1} \mathrm{X}_{\mathrm{n}-3}^{2} \\
& -1.7684 \mathrm{X}_{\mathrm{n}-2} \mathrm{X}_{\mathrm{n}-3}^{2}+1.1800 \mathrm{X}_{\mathrm{n}-2}^{2} \mathrm{X}_{\mathrm{n}-3}
\end{aligned}
$$

These interval extensions were simulated using the initial condition $X_{n-p}=1$ for $p=1,2,3$.

Figure 4 shows the free-run simulation for the system. As well as the Duffing-Ueda oscillator, for Chua's circuit, running different software cause a different orbit in the time series. Figure 5 shows the evolution of the Lower Bound Error. Analyzing the number of iterations that satisfies $\varepsilon=0.001$, the simulation is no longer reliable when $n \geq 545$ for Python, $n \geq 579$ for Matlab and $n \geq 624$ for Octave. For this reason, Matlab represents a $6.24 \%$ greater reliability compared to Python and Octave represents a $14.5 \%$ greater reliability compared to Python. Table 1 shows the reliability summary of the systems studied for the three proposed softwares.

Table 1. Number of iterations that the system is not more reliable according to the simulation criterion given by Eq. 7 .

\begin{tabular}{cccc}
\hline & Python & Matlab & Octave \\
\hline Duffing-Ueda & 4450 & 4471 & 5258 \\
Chua's circuit & 545 & 579 & 624 \\
\hline
\end{tabular}

In addition, it was calculated the time each software takes to process each algorithm. This was used as a method of comparison, since other works Chaves et al. (2006); Unpingco (2008) also use this method for comparison. The algorithm developed in Python was realized with the aid of the NumPy library. And, in each software was simulated a hundred times each algorithm and averaged the time of that hundred times. In addition, the standard deviation was calculated, which indicates how far the data are from the mean. Table 2 shows the results found.

Table 2. Average time of one hundred attempts to execute the proposed algorithm. We have also presented one standard deviation in order to consider the intrinsic fluctuation of time consumption in a computer.

\begin{tabular}{ccc}
\hline & Duffing-Ueda & Chua's circuit \\
\hline Matlab & $0.0425 \pm 0.0179$ & $0.0249 \pm 0.0104$ \\
Python & $1.4719 \pm 0.266$ & $0.3064 \pm 0.0442$ \\
Octave & $3.2808 \pm 0.5919$ & $2.2725 \pm 0.5214$ \\
\hline
\end{tabular}

From Table 2 it is possible to notice that the software Matlab presents a runtime for the same algorithm much smaller than the time presented by Python. And although the Octave presents a greater reliability for the system, the processing time for this software was much higher.

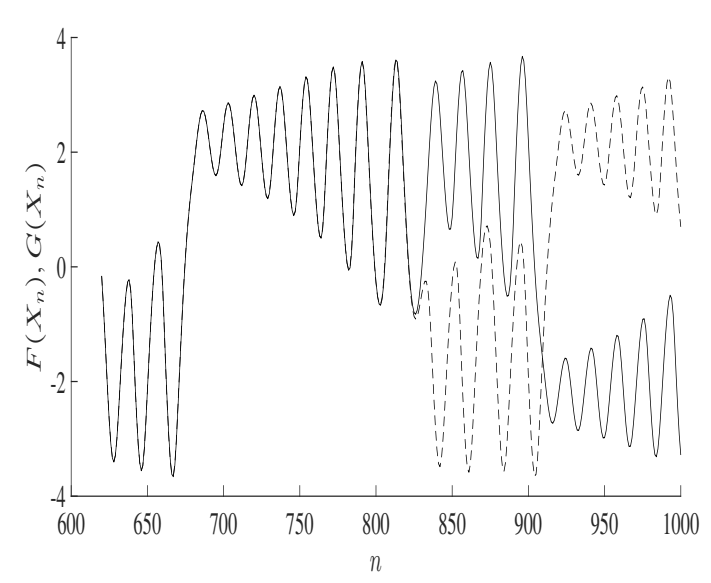

(a) Pseudo-orbits obtained from Python.

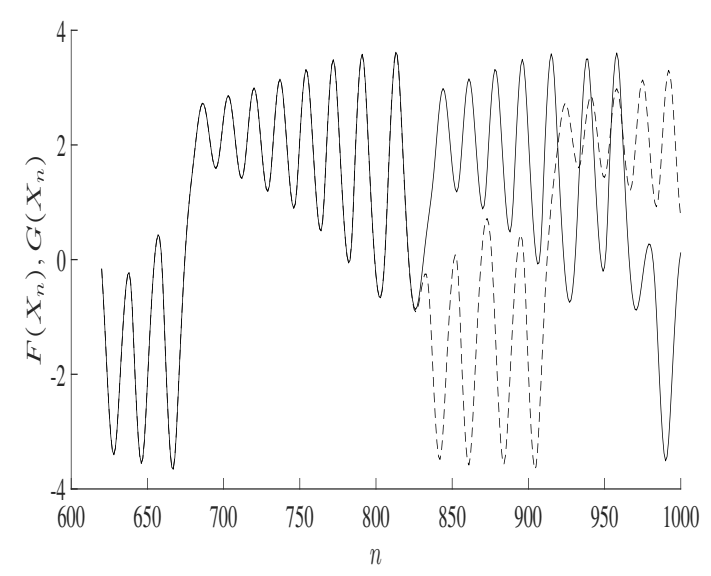

(b) Pseudo-orbits obtained from Matlab

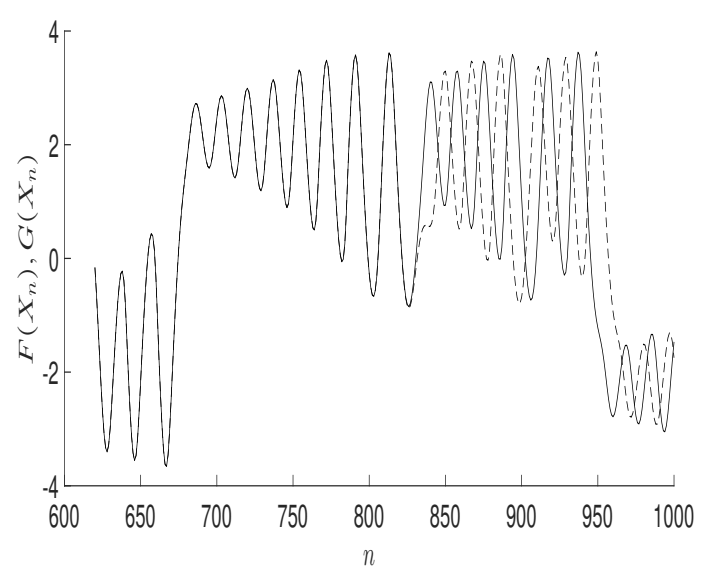

(c) Pseudo-orbits obtained from Octave

Figure 4. Chua's circuit: free-run simulation for the interval extensions of Equations (15) and (16), with results for $F\left(X_{n}\right)(-)$ and $G\left(X_{n}\right)(--)$ and $n$ stands for the number of iterations.

For the Duffing-Ueda oscillator, the processing time of Python is about 50 times greater and Octave about 95 times greater when both are compared with Matlab. Already for Chua's circuit, this time is about 15 and 152 times greater for Python and Octave, respectively. 


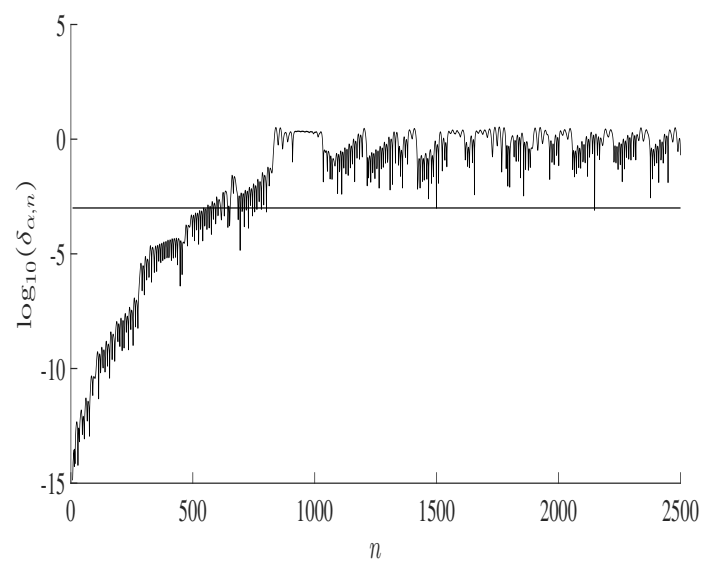

(a) LBE obtained from Python.

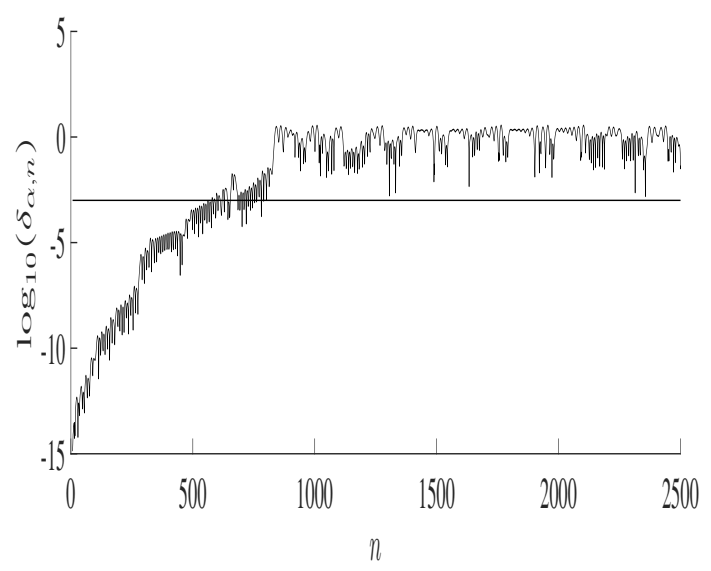

(b) LBE obtained from Matlab

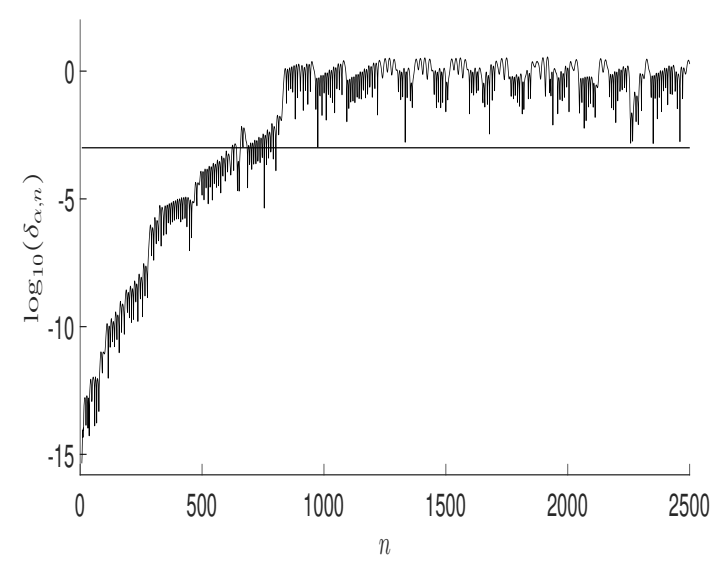

(c) LBE obtained from Octave

Figure 5. Chua's circuit: Evolution of lower bound error $\ell_{\Omega, n}$. The values are plotted using $\log _{10}$.

\section{CONCLUSION}

This work has presented a comparison of the performance of Matlab, Octave and Python in the simulation of dynamical systems. We have adopted the lower bound error as an index. We have also considered the time cost of the simulation. The LBE offer a way to estimate the maximum number of iterations, or a stop criterion, from which the simulation did not present confidence.

Matlab and Octave work with the functions built into the software itself, while in Python it's called via front-end, as with NumPy. Due to these factors Matlab is significantly faster for the calculation of the proposed algorithm. But, Octave presented a slightly higher simulation reliability for this application. The Matlab processing time for this application was the shortest. Python, on the other hand, has been the slowest and it has been intermediate regarding the maximum number of iterations. For future works, we have planned to compare these results using the Ocean Code initiative of IEEE El-Hawary (2018).

\section{REFERENCES}

Aguirre, L.A. and Billings, S. (1994). Validating identified nonlinear models with chaotic dynamics. International Journal of Bifurcation and Chaos, 4(01), 109-125.

Aguirre, L.A. (1997). Recovering map static nonlinearities from chaotic data using dynamical models. Physica D: Nonlinear Phenomena, 100(1-2), 41-57.

Billings, S.A. (2013). Nonlinear system identification: NARMAX methods in the time, frequency, and spatiotemporal domains. John Wiley \& Sons.

Chaves, J.C., Nehrbass, J., Guilfoos, B., Gardiner, J., Ahalt, S., Krishnamurthy, A., Unpingco, J., Chalker, A., Warnock, A., and Samsi, S. (2006). Octave and python: High-level scripting languages productivity and performance evaluation. Proceedings - HPCMP Users Group Conference, 429-434.

Chen, S. and Billings, S.A. (1989). Representations of non-linear systems: the NARMAX model. International Journal of Control, 49(3), 1013-1032.

Chua, L.O., Wu, C.W., Huang, A., and Zhong, G.Q. (1993). A universal circuit for studying and generating chaos. i. routes to chaos. IEEE Transactions on Circuits and Systems I: Fundamental Theory and Applications, 40(10), 732-744.

de la Fraga, L.G., Tlelo-Cuautle, E., and Azucena, A.D.P. (2017). On the execution time of a computational intensive application in scripting languages. In 2017 5th International Conference in Software Engineering Research and Innovation (CONISOFT). IEEE.

El-Hawary, M. (2018). Lotfi zadeh, the 2018 flagship conference, and code ocean [editorial]. IEEE Systems, Man, and Cybernetics Magazine, 4(3), 3-3.

Galias, Z. (2013). The dangers of rounding errors for simulations and analysis of nonlinear circuits and systems? and how to avoid them. IEEE Circuits and Systems Magazine, 13(3), 35-52.

Gilmore, R. and Lefranc, M. (2012). The topology of chaos: Alice in stretch and squeezeland. John Wiley \& Sons.

Guedes, P.F.S., Peixoto, M.L.C., Barbosa, A.M., Martins, S.A.M., and Nepomuceno, E.G. (2017). The lower bound error for polynomial NARMAX using an arbitrary number of natural interval extensions. In DINCON 2017 - Conferência Brasileira de Dinâmica, Controle e Aplicações.

Hammel, S.M., Yorke, J.A., and Grebogi, C. (1987). Do numerical orbits of chaotic dynamical processes represent true orbits? Journal of Complexity, 3(2), 136-145.

IEEE Spectrum (2018). IEEE spectrum ranking. 
Junior, W.R.L., Barbosa, M.S., Nepomuceno, E.G., and Martins, S.A.M. (2017). Confiabilidade numérica na simulaçao de sistemas caóticos utilizando Matlab e $\mathrm{C}++$. Revista de Engenharias da Faculdade Salesiana, $6,2-9$.

Liao, S. (2009). On the reliability of computed chaotic solutions of non-linear differential equations. Tellus, Series A: Dynamic Meteorology and Oceanography, 61(4), 550-564.

Ljung, L., Singh, R., Zhang, Q., Lindskog, P., and Iouditski, A. (2009). Developments in the mathworks system identification toolbox. IFAC Proceedings Volumes, $42(10), 522-527$.

Lofberg, J. (2004). Yalmip: A toolbox for modeling and optimization in matlab. In 2004 IEEE international conference on robotics and automation (IEEE Cat. No. 04CH37508), 284-289. IEEE.

Lozi, R. (2013). Can we trust in numerical computations of chaotic solutions of dynamical systems? In Topology and Dynamics of Chaos: In Celebration of Robert Gilmore's 70th Birthday, 63-98. World Scientific.

May, R.M. (1976). Simple mathematical models with very complicated dynamics. Nature, 261(5560), 459.

Mendes, E.M.A.M. and Nepomuceno, E.G. (2016). A Very Simple Method to Calculate the (Positive) Largest Lyapunov Exponent Using Interval Extensions. International Journal of Bifurcation and Chaos, 26(13), 1650226.

Moore, R.E., Kearfott, R.B., and Cloud, M.J. (2009). Introduction to interval analysis. SIAM.

Nepomuceno, E. and Martins, S. (2016). A lower bound error for free-run simulation of the polynomial NARMAX. Systems Science \& Control Engineering, 4(1), 50-58.

Nepomuceno, E., Martins, S., Amaral, G., and Riveret, R. (2017). On the lower bound error for discrete maps using associative property. Systems Science 8 Control Engineering, 5(1), 462-473.

Nepomuceno, E.G. (2014). Convergence of recursive functions on computers. The Journal of Engineering, 2014(10), 560-562.

Nepomuceno, E.G. and Mendes, E.M. (2017). On the analysis of pseudo-orbits of continuous chaotic nonlinear systems simulated using discretization schemes in a digital computer. Chaos, Solitons and Fractals, 95, 2132.

Qin, S. and Liao, S. (2018). Influence of round-off errors on the reliability of numerical simulations of chaotic dynamic systems. Journal of Applied Nonlinear Dynamics, $7(2), 197-204$.

Sauer, T., Grebogi, C., and Yorke, J.A. (1997). How long do numerical chaotic solutions remain valid? Physical Review Letters, 79(1), 59-62.

Unpingco, J. (2008). Some comparative benchmarks for linear algebra computations in matlab and scientific python. In 2008 DoD HPCMP Users Group Conference, 503-505.

Unpingco, J. (2008). Some comparative benchmarks for linear algebra computations in MATLAB and scientific Python. 2008 Proceedings of the Department of Defense High Performance Computing Modernization Program: Users Group Conference - Solving the Hard Problems, 503-505. 